\title{
Research on Transmission Line Loss and Carrying Current Based on Temperature Power Flow Model
}

Lei LUO ${ }^{a^{*}}$, Xingong CHENG $^{b}$, Xiju ZONG $^{c}$, Wen WEI ${ }^{d}$, Chao WANG ${ }^{\mathrm{e}}$

School of Electrical Engineering, University of Jinan, Jinan, 250022, China;

a*luoleig@163.com, bcse_cxg@ujn.edu.cn,

ccse_zongxj@ujn.edu.cn, ${ }^{\mathrm{d}}$ postweiwen@163.com, ${ }^{\mathrm{e}}$ wangchaoxb@163.com

\section{Keywords: Power Flow; Line Loss; Temperature; Carrying Current}

\begin{abstract}
In conventional power flow calculation, the lines resistances are always assumed to be invariable, which does not conform to the actual. Resistances of transmission lines are changed with changes of external environment and power distribution. In this paper, a model of power flow calculation considering temperature is presented. Temperatures and resistances of branches are linked with the modified power flow equations in this model. At the same time, the maximum current-carrying capacity is also analyzed and temperature can be gained by the algorithm to detect current. Through two kinds of power flow algorithm are compared and analyzed, the results show temperature of conductor has a very significant impact on the power flow calculation which should be paid attention to.
\end{abstract}

\section{Introduction}

Power flow calculation is based on a given system network structure and operating conditions to determine the running state of the whole system: mainly is the node voltage (amplitude and phase), power distribution and power loss [1]. Electro-thermal coupling research showed that temperature and resistance in transmission lines follow the outside temperature, light intensity, wind speed and other environmental factors. At the same time, temperature changes produced by electro-thermal will influence the power system loss and flow calculation results. Although the resistance of the branch is sensitive to the temperature, the resistance of the system is always treated as normal. Not promptly adjusting branch resistances may produce significant errors under high load conditions----up to 10\% for the total system loss, $30 \%$ for the branch alone [2] [3].

In [4] [5], the concept and application of electro-thermal coordination are presented, and the coupling and conversion between electric and heat are considered, which provides a basis for further study on power flow calculation. In [6], a dynamic electro-thermal coordination method is presented to calculate the dynamic power flow. The algorithm considered temperature changes of power components to achieve the purpose of transformation between electricity and heat. The algorithm is relatively complicated and is not suitable for the large scale, multi-coupling and nonlinear power networks. The relationship between the temperature rise and the impedance of the power system equipment is given in the paper [7-10], which provides a basis for establishing the generalized electric power system electric heating model.

In this paper, a new model considering temperature is established by improving the conventional method. The generalized model of electric power equipment is taken into account. The temperature is one of the state variables to establish temperature load flow model. Through given system thermal and environmental data in addition to power injections, the model links these relations with the conventional power flow by constructing a series of equations for branch resistance, loss and temperature. At the same time, this paper also estimates electric current of the transmission line by temperature to avoid transmitting electric current exceeding the allowable values, causing unnecessary operation. Finally, the simulation demonstrates the validity and feasibility of the proposed method. The case study and comparison of the calculations by two methods show that there exist significantly differences of the results. 


\section{Relationship between Temperature and Conductor Resistance}

Fig. 1 is the diagram of thermal resistance model of electrical components. This model simulates the thermal response of power equipment [9] [10]. In the thermal resistance model, the temperature of the equipment is proportional to the loss of the equipment. The ratio of the temperature rise to loss in the steady state is the thermal resistance $R_{\theta}$.

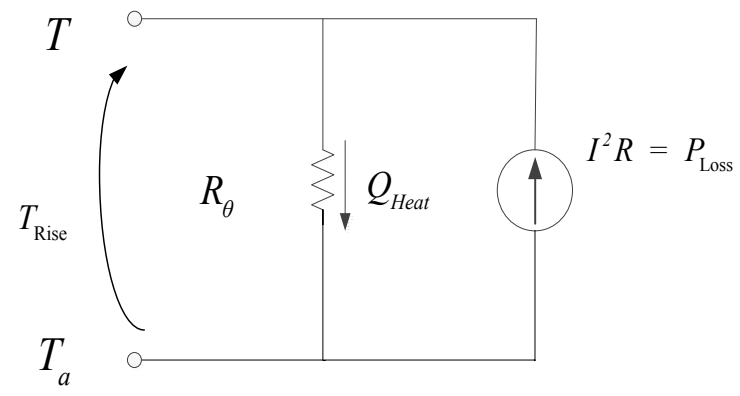

Fig. 1 Thermal resistance model of electric element

For the resistive loss, the temperature rise is proportional to the heat generated [10],

$\frac{T_{\text {Rise }}}{P_{\text {Loss }}}=\frac{T_{\text {RatedRise }}}{P_{\text {RatedLoss }}}=R_{\theta}$

In which $T_{\text {Rise }}$ is temperature rise above the ambient, $P_{\text {Loss }}$ is all the loss within the device, $T_{\text {RatedRise }}$ is reference device temperature rise, $P_{\text {RatedLoss }}$ is corresponding reference loss.

Conductor temperature $T$ is equal to the ambient temperature $T_{a}$ and temperature $\operatorname{rise} T_{\text {Rise }}$, namely, $T=T_{a}+T_{\text {Rise }}$

Therefore, by rearranging (1) (2),

$$
T=T_{a}+\left(\frac{P_{\text {Loss }}}{P_{\text {RatedLoss }}}\right) T_{\text {RatedRise }}
$$

Assuming the transmission line is the ideal uniform material, the temperature of the transmission line is equal to the temperature of the surrounding without the current. In the steady state, the heat transfer of transmission line is determined by the heat balance equation [3],

$$
Q_{j}+Q_{s}=Q_{r}+Q_{c}
$$

Where $Q_{j}$ is Joule loss, $Q_{s}$ is heat absorption from sunshine, $Q_{r}$ is radiation heat transfer, $Q_{c}$ is convective heat transfer.

Joule losses are computed from

$Q_{j}=I^{2} R_{c}$

In which $I$ is the conductor current and $R_{c}$ is the unitary resistance. The conductor resistance is a function of temperature, as follows [11]:

$$
R_{c}=R_{\mathrm{Ref}} \times \frac{T+T_{F}}{T_{\mathrm{Ref}}+T_{F}}
$$

In which $R_{c}$ is conductor resistance, $T$ is conductor temperature; $R_{\mathrm{Ref}}$ is conductor resistance at temperature $T_{\text {Ref }} ; T_{\text {Ref }}$ is reference temperature; $T_{F}$ is temperature constant.

The conductor is also heated by solar radiation, given by

$Q_{s}=W_{s} d_{c}$

In which $W_{s}$ stands for the incident solar energy and $d_{c}$ is the conductor diameter.

Convection and radiation heat emission can be computed by means of empirical expressions. 


$$
\begin{aligned}
& Q_{c}=S \frac{5.727 \sqrt{p v}}{\left(T_{a}+273.15\right)^{0.123} \sqrt{d_{c}}}\left(T_{c}-T_{a}\right) \\
& Q_{r}=17.8204 E d_{c}\left[\left(\frac{T_{c}+273.15}{100}\right)^{4}\left(\frac{T_{a}+273.15}{100}\right)^{4}\right]
\end{aligned}
$$

Where $S$ is conductor surface, $p$ is atmospheric pressure, $v$ is wind speed, $E$ is emissivity constant.

$Q_{r}, Q_{c}$ can be approximated as linear functions of the conductor temperature under certain environmental condition, the above factors can be expressed as [3],

$$
\begin{aligned}
& Q_{c} \approx A_{c}\left(T-T_{a}\right) \\
& Q_{r} \approx A_{r}\left(T-T_{a}\right)
\end{aligned}
$$

In which $A_{c}, A_{r}$ are the convective transfer coefficient and the thermal radiation heat transfer coefficient respectively. They are affected by the transmission line material, environmental factors, such as wind velocity, ambient temperature, material emissivity, radiating surface area.

Because all the heat $Q_{j}+Q_{s}$ are produced within the line, $1 /\left(A_{c}+A_{r}\right)$ can be analogous to $R_{\theta}$ in the thermal model. Using $Q_{j}=P_{\text {Loss }}$, this allows a modified version of (2) to be written for conductors:

$$
T=T_{\mathrm{a}}+\left(P_{\text {Loss }}+Q_{s}\right) \times \frac{1}{A_{c}+A_{r}}
$$

\section{The Power Flow Model and Temperature Change Process}

The power flow algorithm considering temperature is like the conventional algorithm, but is different from the coordination electro-thermal. The system is assumed to operate stably.

The power flow model considering the temperature can be expressed as:

$f(X, Y, Z)=0$

Where $X$ stands for control variables, such as active power, reactive power, etc. $Y$ is the state variables of the system, such as voltage magnitude, phase angle, etc. $Z$ indicates the relevant parameters, such as component resistance, reactance, load, etc.

For a system with $\mathrm{K}$ buses, $\mathrm{N}$ of which are PQ buses, and L dependent temperature of the branches. There are K-lunknown voltage angles, $\mathrm{N}$ unknown voltage magnitudes and $\mathrm{L}$ unknown temperatures. In addition to the conventional state variable $V$ and $\theta$, the state variable $T$ is required as the temperature value of each dependent temperature branch. State vector generates form $X=[\theta, V, T]^{\mathrm{T}}$.

The Jacobian matrix is augmented as follows

$$
\left[\begin{array}{c}
\Delta P \\
\Delta Q \\
\Delta E
\end{array}\right]_{K \times 1}=\left[\begin{array}{ccc}
\frac{\partial \Delta P}{\partial \theta} & \frac{\partial \Delta P}{\partial V} V & \frac{\partial \Delta P}{\partial T} \\
\frac{\partial \Delta Q}{\partial \theta} & \frac{\partial \Delta Q}{\partial V} V & \frac{\partial \Delta Q}{\partial T} \\
\frac{\partial \Delta E}{\partial \theta} & \frac{\partial \Delta E}{\partial V} V & \frac{\partial \Delta E}{\partial T}
\end{array}\right]_{K \times K}\left[\begin{array}{c}
\Delta \theta \\
\frac{\Delta V}{V} \\
\Delta T
\end{array}\right]_{K \times 1}
$$

Where mismatches may be defined as the difference between the expected and calculated equation values:

$$
\begin{aligned}
\Delta P_{i} & =\left(P_{\text {Gen.i }}-P_{\text {Load.i }}\right)-P_{i}(\theta, V, T) \\
\Delta Q_{i} & =\left(Q_{\text {Gen }, \mathrm{i}}-Q_{\text {Load,i }}\right)-Q_{i}(\theta, V, T)
\end{aligned}
$$




$$
\Delta E_{i}=0-E_{i}(\theta, V, T)
$$

Because the conductor resistance is a function of temperature, we cannot directly obtain the partial derivatives of $T$. Therefore, the chain rule must be used. For example:

$$
\frac{\partial \Delta P_{i}}{\partial T_{k n}}=\frac{\partial \Delta P_{i}}{\partial g_{k n}} \times \frac{\partial g_{k n}}{\partial R_{k n}} \times \frac{\partial R_{k n}}{\partial T_{k n}}+\frac{\partial \Delta P_{i}}{\partial b_{k n}} \times \frac{\partial b_{k n}}{\partial R_{k n}} \times \frac{\partial R_{k n}}{\partial T_{k n}}
$$

Where $T_{k n}, g_{k n}, R_{k n}$ and $b_{k n}$ are line temperature, conductance, resistance and susceptance from bus $k$ to $n$, respectively.

Once the Jacobian matrix is defined, iterative equations become:

$\left[\begin{array}{l}\theta^{v+1} \\ V^{v+1} \\ T^{v+1}\end{array}\right]=\left[\begin{array}{l}\theta^{v} \\ V^{v} \\ T^{v}\end{array}\right]+J\left(\theta^{v}, V^{v}, T^{v}\right)^{-1} \times\left[\begin{array}{c}\Delta P^{v} \\ \Delta Q^{v} \\ \Delta E^{v}\end{array}\right]$

General convergence criterion:

$$
\max _{i}\left|\Delta P_{i}^{v}\right|<\varepsilon, \max _{i}\left|\Delta Q_{i}^{v}\right|<\varepsilon, \max _{i}\left|\Delta E_{i}^{v}\right|<\varepsilon
$$

In which $v$ is the iteration number. Flow process is illustrated in Fig. 2

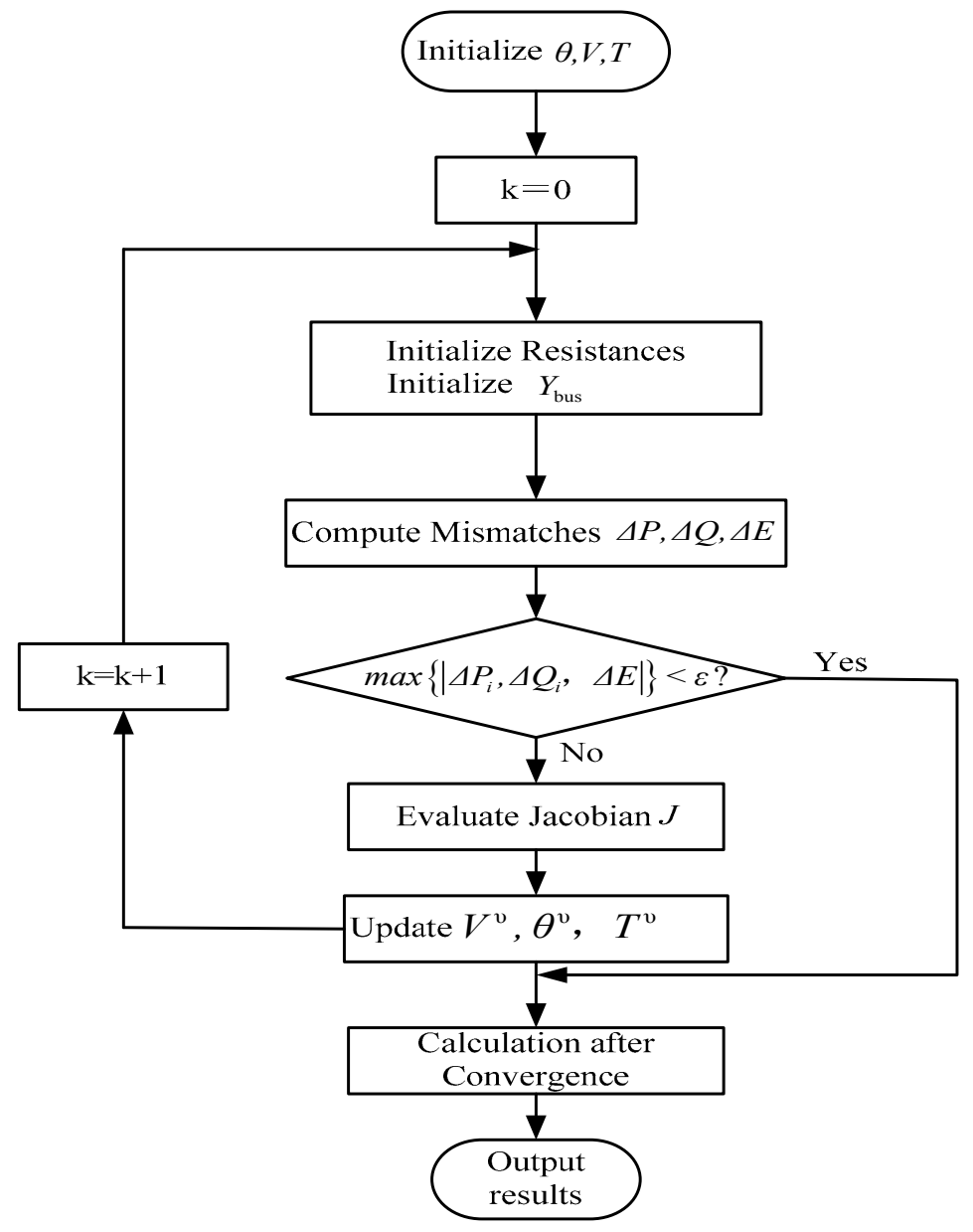

Fig. 2 Flow process diagram of program

\section{Carrying Current Estimated by Temperature}

When the actual power system is running, the temperature rise of the transmission line is unbalanced because of the difference of the load current and the change rate of the transmission line.

Under any initial conditions, when the temperature of the transmission line is unchanged, the equilibrium state is reached. When a certain balance of current-carrying capacity corresponds to the 
allowable temperature of the transmission line, the current-carrying capacity is the allowable flow of the transmission line [11].

$$
Q_{s}(\infty)+I_{\max }^{2} \times R\left(T_{\max }\right)=Q_{c}\left(T_{\max }\right)+Q_{r}\left(T_{\max }\right)
$$

Thus, the load flow permitted by heat in the transmission line can be expressed as:

$$
I_{\text {max }}=\sqrt{\frac{Q_{c}\left(T_{\max }\right)+Q_{r}\left(T_{\max }\right)-Q_{s}(\infty)}{R\left(T_{\max }\right)}}
$$

The current technical regulation is set in the conservative meteorological conditions (such as higher ambient temperature, strong sunshine, lower wind speed, etc.). The maximum allowable temperature of the long (or short) heating of the transmission line is $T_{\max }$ and the result of (22) is conservative. From the long-term maximum allowable current concept can be seen, the largest current determination is in consideration of single transmission line. Of course, it can be conveniently determined the maximum current-carrying capacity under different conditions according to the different settings of the scene. For a long time, the operation of the actual power system is basically based on the method.

The power flow of transmission line can be expressed as (ignore susceptance)

$$
\begin{aligned}
& P_{j k}=U_{j}^{2} g_{j k}(T)-U_{j} U_{k}\left[g_{j k}(T) \cos \theta_{j k}-b_{j k}(T) \sin \theta_{j k}\right] \\
& Q_{j k}=U_{j}^{2} b_{j k}(T)-U_{j} U_{k}\left[g_{j k}(T) \sin \theta_{j k}-b_{j k}(T) \cos \theta_{j k}\right]
\end{aligned}
$$

The current through the transmission line is:

$$
I_{j k}=\sqrt{\frac{P_{j k}^{2}+Q_{j k}^{2}}{U_{j}^{2}}}
$$

In which $U_{j}, U_{k}$ are respectively for the bus $j$ and $k$ voltage amplitude; $g_{j k}, b_{j k}$ are conductance and susceptance from bus $j$ to $k$, respectively; $\theta_{j k}$ is the difference of voltage phase angles from bus $j$ to $k$. It is known that $g_{j k}=R_{j k} /\left(X_{j k}^{2}+R_{j k}^{2}\right)\left(R_{j k}\right.$ and $X_{j k}$ are resistance and reactance respectively.) and $b_{j k}=-X_{j k} /\left(X_{j k}^{2}+R_{j k}^{2}\right)$ are associated with the resistance component. Therefore, and the power flow is also the change with the temperature.

When a disturbance (load change, power generation mode) occurs, the temperature rise of transmission line is different. From this, the process of temperature rise should be integrated from the whole system, not being isolated. The limit of the power flow is monitored by the actual temperature and the security of the system can be guaranteed in case of avoiding excessive current-carrying capacity.

\section{Simulation}

The New England IEEE39 bus system is publicly available as an example. The system consists of 10 generators, 19 loads and 46 transmission lines. System base volume and temperature were 100MVA and $100^{\circ} \mathrm{C}$, respectively. All the parameters of the grid are converted to p.u. and all line conductors are using hard-drawn aluminum wire $\left(T_{F}=228.1^{\circ} \mathrm{C}\right)$. Assuming that all the line conductors are considered the temperature loss of the branch, the wind speed and other environmental factors are negligible. In order to verify the correctness of the method proposed, the case is simulated in the environment of Matlab. Assuming that the initial temperature of all line conductors is set to $25^{\circ} \mathrm{C}$, the calculation results of conventional power flow (Newton-Raphson method) algorithm and considering temperature power flow algorithm are given, respectively. Computer configurations are: the processor Intel (R) Pentium (R) G2030 CPU @ 3.00GHz, RAM for 4GBytes, the system for Windows 7. 
In Tab.1, from a running time comparison of two algorithms can be seen, considering temperature plow flow algorithm is longer than conventional algorithm in running time because the new model improves the requirements of the convergence and increases running time.

Tab. 1 Running time comparison

\begin{tabular}{cllll}
\hline Algorithm & \multicolumn{2}{l}{ Conventional PF } & \multicolumn{3}{c}{$\begin{array}{c}\text { Considering } \\
\text { temperature PF }\end{array}$} \\
\hline Iterations & 1 & \multicolumn{3}{c}{4} \\
Setup & $7.4 \mathrm{~ms}$ & $(11.92 \%)$ & $6.8 \mathrm{~ms}$ & $(5.23 \%)$ \\
Calculating Y Bus & $5.1 \mathrm{~ms}$ & $(8.16 \%)$ & $10.3 \mathrm{~ms}$ & $(7.85 \%)$ \\
Calculating Jacobian & $39.4 \mathrm{~ms}$ & $(63.26 \%)$ & $89.5 \mathrm{~ms}$ & $(68.44 \%)$ \\
Calculating Mismatches & $0.7 \mathrm{~ms}$ & $(1.16 \%)$ & $3.2 \mathrm{~ms}$ & $(2.43 \%)$ \\
Calculating Updates & $1.6 \mathrm{~ms}$ & $(2.59 \%)$ & $5.3 \mathrm{~ms}$ & $(4.07 \%)$ \\
Overhead & $8.1 \mathrm{~ms}$ & $(12.91 \%)$ & $12.2 \mathrm{~ms}$ & $(9.34 \%)$ \\
Updating & $0 \mathrm{~ms}$ & & $3.5 \mathrm{~ms}$ & $(2.65 \%)$ \\
Temperatures & & & & \\
Total & $62.4 \mathrm{~ms}$ & $(100.00 \%)$ & $130.8 \mathrm{~ms}$ & $(100.00 \%)$ \\
\hline
\end{tabular}

The line loss can be observed directly by the visual observation of the two models in Fig. 3. The temperature is different in different branch number. However, some branches are sensitive, such as branch 6-11, 16-19 and so on. The difference of line loss calculated between conventional method and considering temperature algorithm to the loss of conventional calculation is defined as the relative loss ratio. By calculating, the total loss of conventional power flow is $43.641 \mathrm{MVA}$, considering temperature power flow is $44.953 \mathrm{MVA}$. The total line loss of considering temperature power flow is up to $3.01 \%$ higher than that of conventional method. However, for some temperature sensitive transmission lines, such as branch 16-19 reached $4.90 \%$, line loss can be seen significantly influenced by temperature factor.

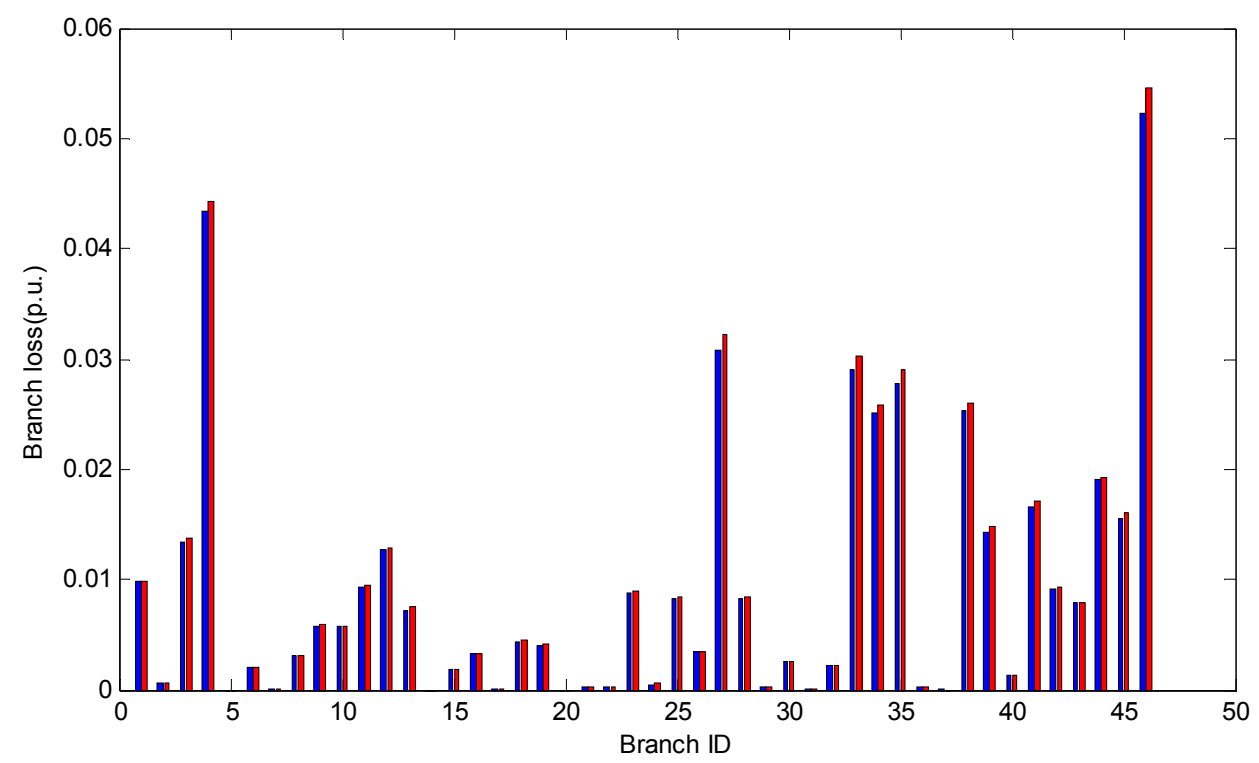

Fig. 3 Comparison of transmission line loss

The assumptions that the external environment temperatures are $5^{\circ} \mathrm{C}, 15^{\circ} \mathrm{C}, 25^{\circ} \mathrm{C}, 35^{\circ} \mathrm{C}$ are used in the cases. With the increase of temperature, the relative loss ratios of some branches are getting higher and higher in Fig.4. The maximum can reach 6.49\%, for the additional loss of power system, which has reached large value. The temperature sensitivity of different branches in power network is different, but the sensitivity of each branch to temperature is determined in a given network. 


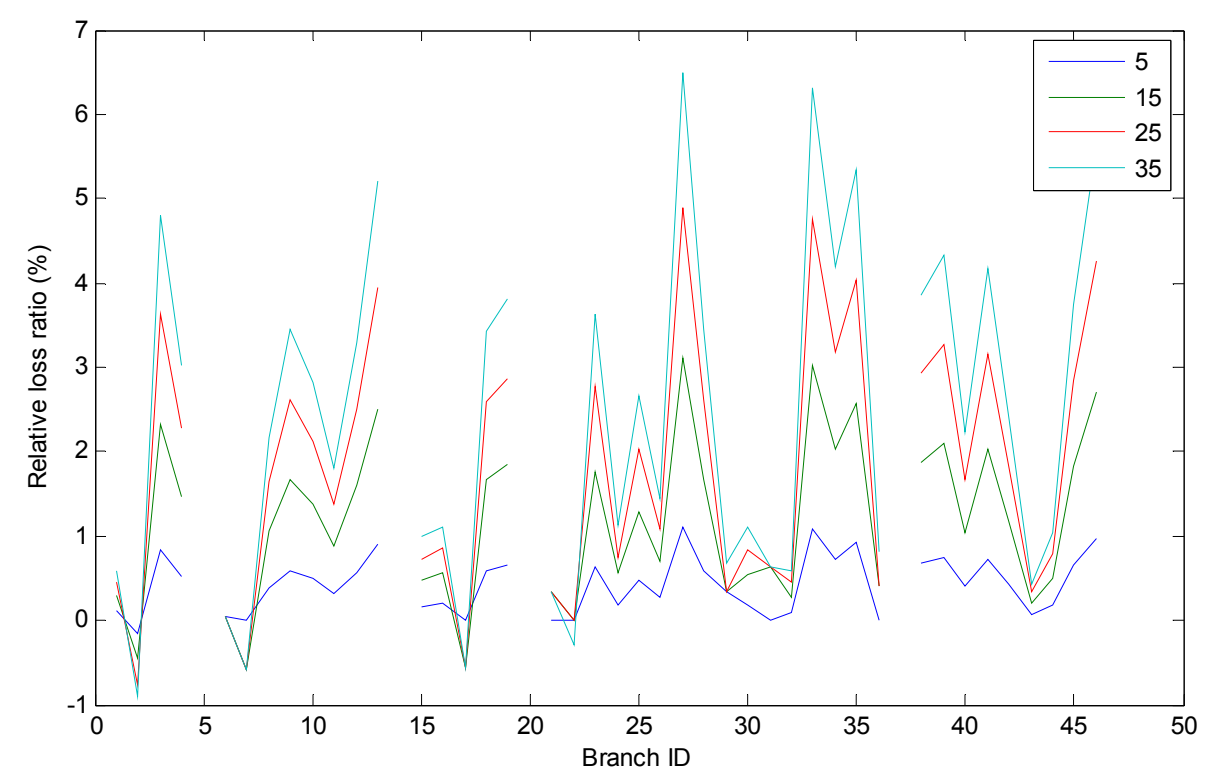

Fig. 4 Relative loss ratio of branch under different environment temperature

The sensitive branch to the electric heat can be obtained by considering temperature power flow model, such as branch 19-33. There exist 642.8A and 653.1A when ambient temperatures are at $15^{\circ} \mathrm{C}$ and $35^{\circ} \mathrm{C}$ respectively in Fig.5. Thus temperature values obtained from the lines are $22.4^{0} \mathrm{C}, 51.8^{0} \mathrm{C}$ respectively. The loss of the corresponding transmission line can be obtained by the power flow calculation, so the current-carrying capacity can be determined by temperature. As long as the temperature does not go beyond the allowable temperature limits, the line will be safe in theory.
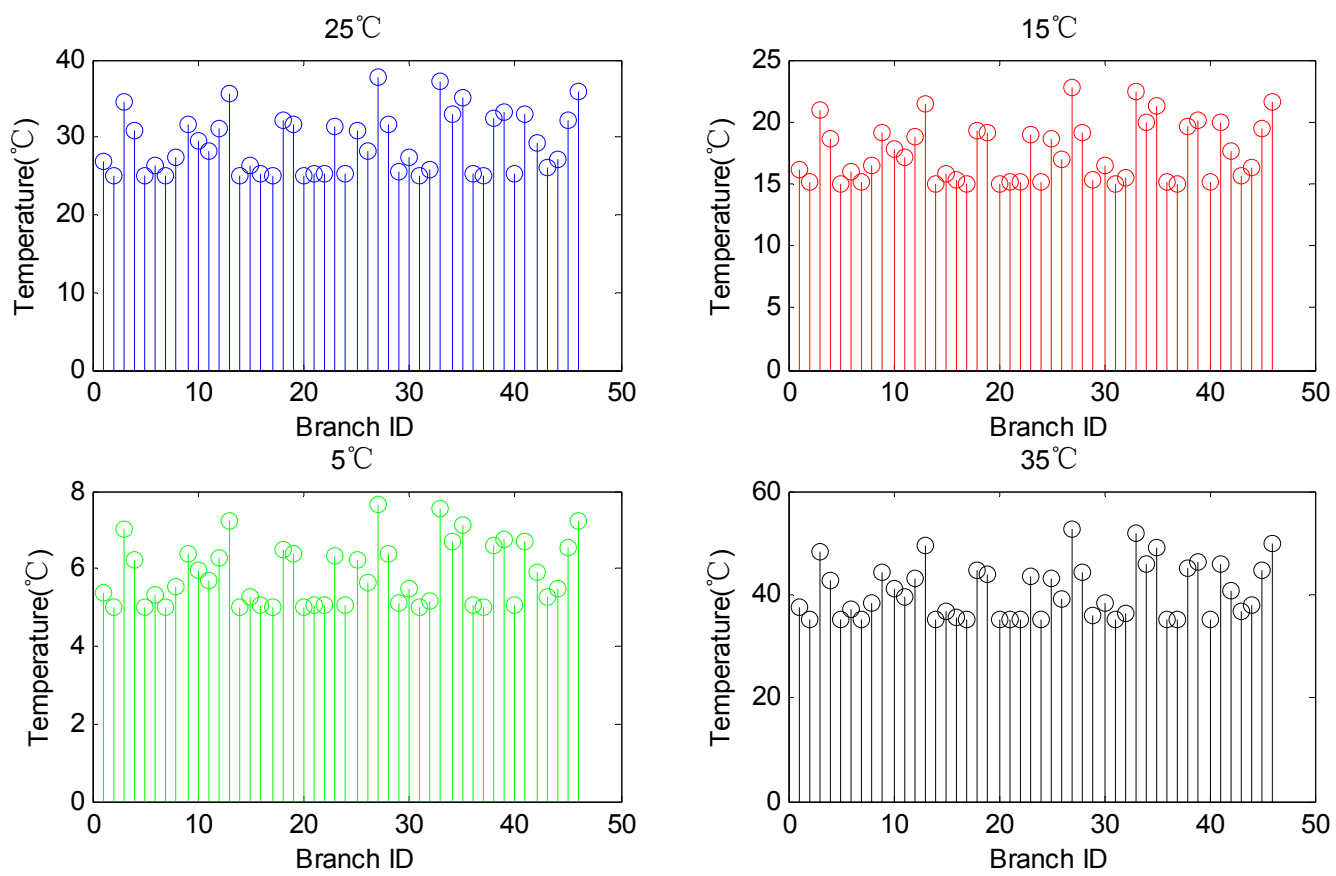

Fig. 6 Branch temperature under different environment temperature

\section{Conclusion}

Temperature produced by electro-thermal causes the change of transmission components and electrical characteristics. Considering temperature power flow model provides an idea for the electro-thermal analysis of power flow. The numerical example shows that temperature has some effect on the power flow distribution of power system. In some branches, the loss is obvious, so the electric heating factor is considered in the power flow analysis necessarily. Through the new power 
flow model, the transmission current-carrying capacity can be determined by temperature and then it can make a judgment to power system decision-making and predict, improving the security and stability of power system.

\section{Acknowledgement}

In this paper, this work was sponsored by National Natural Science Foundation of China under Grant 11201179. The authors wish to thank the reviewers for their constructive comments and suggestions which have helped to improve the presentation of the paper.

\section{References}

[1] Wang Shouxiang, Liu Yutian. Review of Load Flow Calculation Methods in Power Systems [J]. Shandong Electric Power, 1996(05):8-9.

[2] IEEE Recommended Practice for Industrial and Commercial Power Systems Analysis, IEEE Std. 399, 1997.

[3] J. R. Santos, A. G. Expósito, and F. P. Sánchez, "Assessment of conductor thermal models for grid studies,” IET Gen., Transm., Distrib.,vol. 1, no. 1, pp. 155-161, Jan. 2007.

[4] DAVIS M W. A new thermal rating approach: Thereal-time thermal rating system for strategic overhead conductor transmission lines part I general description and justification of the real time thermal rating system[J].IEEE Trans on Power Apparatus and Systems, 1977, 96(3): 803-809.

[5] DAVIS M W. A new thermal rating approach: the real time thermal rating system for strategic overhead conductor transmission lines part IV daily comparisons of real-time and conventional thermal ratings and establishment of typical annual weather models [J]. IEEE Transon Power Apparatus and Systems, 1980, 99(6):2184-2192.

[6] WANG Meng-xia, HAN Xue-shan, JIANG Zhe, et al.Power flow model and algorithm considering electro-thermal coupling [J]. Automation of Electric Power Systems, 2008, 32(14): 30-34.

[7] Alguacil N, Banakar H, Galiana F D . Electrothermal coordination PartII: case sduties[J]. IEEE Trans. on Power Systems, 2005, 20(2): 1738-1745.

[8] Banakar H, Alguacil N, Galiana F D. Electrothermal coordination PartI : Theory and implementation scheme[J]. IEEE Trans. on Power Systems, 2005, 20(2): 798-805.

[9] IEEE Standard Test Code for Dry-Type Distribution and Power Transformers, IEEE Std. C57.12.91, 2001.ZHANG Hui, HAN Xue-shan, WANG Yan-ling. Analysis on current carraying capacity of overhead lines being operated [J]. Power System Technology, 2008, 32(14):31-35.

[10] IEEE Recommended Practice for Industrial and Commercial Power Systems Analysis, IEEE Std. 399, 1997.

[11] IEEE Standard Test Code for Dry-Type Distribution and Power Transformers, IEEE Std. C57.12.91, 2001. 